\title{
Multireference Perturbation Theory Combined with PCM and RISM Solvation Models: A Benchmark Study for Chemical Energetics Supplementary material
}

\author{
Masaaki Saitow, ${ }^{* \dagger}$ Keisuke Hori,${ }^{\dagger}$ Ayaka Yoshikawa, ${ }^{\ddagger}$ Ryosuke \\ Y. Shimizu, ${ }^{\dagger}$ Daisuke Yokogawa ${ }^{\amalg}$ and Takeshi Yanai ${ }^{\dagger \S}$ \\ $\dagger$ Department of Chemistry, Graduate School of Science, Nagoya \\ University, Furocho, Chikusa Ward, Nagoya, Aichi 464-8601, Japan \\ $\ddagger$ Department of Chemistry, Nagoya University, Furocho, Chikusa Ward, Nagoya, Aichi 464-8601, Japan \\ $\amalg$ Department of Arts and Sciences, The University of \\ Tokyo, Komaba, Meguro-Ward, Tokyo, 153-8902, Japan \\ $\S$ Institute of Transformative Bio-Molecules (WPI-ITbM), Nagoya \\ University, Furocho, Chikusa Ward, Nagoya, Aichi 464-8601, Japan \\ * email: msaitow514@gmail.com
}


This material contains the MEPs calculated by the IEF-PCM and C-PCM for aniline, the optimized geometries of pyridine, aniline, phenol, $p$-Br-phenol and Cl-benzene which were optimized in both water and in gas-phase, the $\mathrm{S}-\mathrm{T}$ gaps without thermal corrections for the subset of AC12 benchmark set and the $\left\langle S^{2}\right\rangle$ values for the polycarbene systems calculated by the unrestricted DFT methods. The optimized structures of the polycarbene species are also shown. The IEFPCM results in comparison with the C-PCM and RISM counterparts are given.

\section{MOLECULAR ELECTROSTATIC POTENTIAL MAPS OF ANILINE IN WATER}

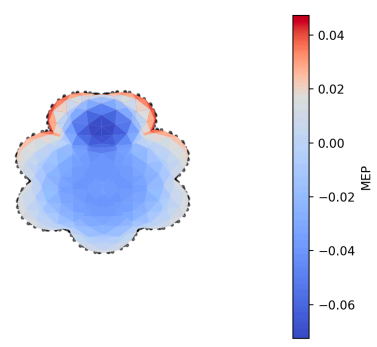

IEFPCM

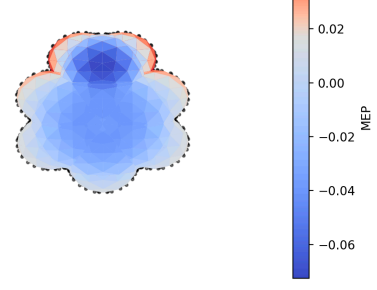

CPCM

FIG. S1. Molecular electrostatic potentials of aniline calculated at the CASSCF level of theory using IEFPCM (left) and C-PCM (right) solvation models.

\section{STRUCTURES OF ORGANIC MOLECULES IN SEC. 4.1}

All the geometries are given in Ångstroms.

\section{A. Structure of Pyridine in gas-phase}

$\begin{array}{lrrr}\mathrm{C} & -1.144550 & -0.723295 & 0.000000 \\ \mathrm{C} & -1.200169 & 0.673706 & 0.000000 \\ \mathrm{C} & 0.000000 & 1.386970 & 0.000000 \\ \mathrm{C} & 1.200169 & 0.673706 & 0.000000 \\ \mathrm{C} & 1.144550 & -0.723295 & 0.000000 \\ \mathrm{~N} & -0.000000 & -1.420768 & -0.000000 \\ \mathrm{H} & 0.000000 & 2.473528 & -0.000000 \\ \mathrm{H} & -2.061119 & -1.310302 & -0.000000 \\ \mathrm{H} & -2.159212 & 1.182849 & -0.000000\end{array}$


H

$\mathrm{H}$

2.159212

2.061119
1.18284

$-1.310302$

$-0.000000$

$-0.000000$

B. Structure of Pyridine in water

C

C

C

C

C

N

H

$\mathrm{H}$

$\mathrm{H}$

H

$\mathrm{H}$

C

C

C

C

C

C

H

$\mathrm{H}$

$\mathrm{H}$

$\mathrm{H}$

$\mathrm{H}$

N

$\mathrm{H}$

$\mathrm{H}$

$\begin{array}{rrr}-1.147513 & -0.722937 & 0.000000 \\ -1.200977 & 0.673611 & 0.000000 \\ 0.000000 & 1.386733 & 0.000000 \\ 1.200977 & 0.673611 & 0.000000 \\ 1.147513 & -0.722937 & 0.000000 \\ -0.000000 & -1.421801 & -0.000000 \\ 0.000000 & 2.472821 & -0.000000 \\ -2.065651 & -1.306736 & -0.000000 \\ -2.159678 & 1.182382 & -0.000000 \\ 2.159678 & 1.182382 & -0.000000 \\ 2.065650 & -1.306736 & -0.000000\end{array}$

C. Structure of aniline in gas-phase

\begin{tabular}{rrrr}
1.884671 & -0.000030 & 0.007329 \\
\hline & -0.221883 & -1.209666 & -0.004238 \\
\hline & -0.939461 & 0.000193 & -0.007676 \\
\hline & 1.173577 & 1.204202 & 0.003337 \\
& 2.970197 & -0.000358 & 0.013952 \\
& 1.707140 & -2.151003 & 0.006646 \\
\hline & -0.762528 & -2.153374 & -0.013127 \\
\hline & -0.762073 & 2.153725 & -0.013368 \\
\hline & -2.707184 & 2.150955 & 0.006995 \\
\hline & -2.793620 & -0.839634 & 0.258266 \\
\hline & -2.793630 & 0.839721 & 0.257609
\end{tabular}


D. Structure of aniline in water

$\begin{array}{lrrr}\text { C } & 1.886573 & -0.000014 & 0.006407 \\ \mathrm{C} & 1.174094 & -1.205530 & 0.003167 \\ \mathrm{C} & -0.222304 & -1.211152 & -0.003974 \\ \mathrm{C} & -0.941495 & 0.000161 & -0.007999 \\ \mathrm{C} & -0.222308 & 1.211197 & -0.004318 \\ \mathrm{C} & 1.174318 & 1.205385 & 0.003358 \\ \mathrm{H} & 2.972281 & -0.000316 & 0.013239 \\ \mathrm{H} & 1.707632 & -2.152423 & 0.008590 \\ \mathrm{H} & -0.763104 & -2.154461 & -0.007158 \\ \mathrm{H} & -0.762720 & 2.154723 & -0.007904 \\ \mathrm{H} & 1.707614 & 2.152408 & 0.008821 \\ \mathrm{~N} & -2.339198 & 0.000128 & -0.076816 \\ \mathrm{H} & -2.790246 & -0.837487 & 0.269578 \\ \mathrm{H} & -2.790331 & 0.836377 & 0.272704\end{array}$

E. Structure of phenol in gas-phase

$\begin{array}{lrrr}\text { C } & -1.134146 & 1.220690 & -0.000004 \\ \text { C } & 0.264001 & 1.201515 & -0.000027 \\ \text { C } & 0.940421 & -0.023685 & 0.000004 \\ \text { C } & 0.222175 & -1.225077 & 0.000019 \\ \text { C } & -1.172477 & -1.192450 & -0.000003 \\ \text { C } & -1.860009 & 0.027138 & 0.000010 \\ \text { H } & -1.652528 & 2.175457 & -0.000004 \\ \text { H } & 0.824873 & 2.134123 & -0.000063 \\ \text { H } & 0.766711 & -2.163889 & 0.000053 \\ \text { H } & -1.725577 & -2.127563 & 0.000022 \\ \text { H } & -2.945415 & 0.045385 & 0.000032 \\ \text { O } & 2.309441 & -0.110808 & -0.000095 \\ \text { H } & 2.696614 & 0.774172 & 0.000729\end{array}$


F. Structure of phenol in water

$\begin{array}{lrrr}\text { C } & -1.131557 & 1.222893 & -0.000000 \\ \mathrm{C} & 0.266959 & 1.203157 & 0.000000 \\ \mathrm{C} & 0.940322 & -0.025079 & 0.000000 \\ \mathrm{C} & 0.220401 & -1.226225 & 0.000000 \\ \mathrm{C} & -1.176300 & -1.192077 & -0.000000 \\ \mathrm{C} & -1.861064 & 0.029285 & 0.000000 \\ \mathrm{H} & -1.648551 & 2.178581 & -0.000000 \\ \mathrm{H} & 0.830308 & 2.133229 & -0.000000 \\ \mathrm{H} & 0.758639 & -2.169238 & -0.000000 \\ \mathrm{H} & -1.730361 & -2.126830 & -0.000000 \\ \mathrm{H} & -2.946653 & 0.050094 & -0.000000 \\ \mathrm{O} & 2.310074 & -0.113551 & -0.000000 \\ \mathrm{H} & 2.703468 & 0.770842 & -0.000000\end{array}$

G. Structure of $p$-Br-phenol in gas-phase

$\begin{array}{lrrr}\mathrm{C} & -1.777241 & -1.200533 & 0.000000 \\ \mathrm{C} & -2.472448 & 0.013964 & -0.000000 \\ \mathrm{C} & -1.768366 & 1.223194 & 0.000000 \\ \mathrm{C} & -0.373808 & 1.216617 & 0.000000 \\ \mathrm{C} & 0.313547 & 0.001526 & -0.000000 \\ \mathrm{C} & -0.379967 & -1.207234 & 0.000000 \\ \mathrm{H} & -2.319387 & -2.143654 & 0.000000 \\ \mathrm{H} & -2.319553 & 2.157801 & 0.000000 \\ \mathrm{H} & 0.173067 & 2.153204 & 0.000000 \\ \mathrm{H} & 0.158880 & -2.148401 & 0.000000 \\ \mathrm{O} & -3.839789 & 0.085245 & 0.000000 \\ \mathrm{H} & -4.221098 & -0.802411 & 0.000000 \\ \mathrm{Br} & 2.228460 & -0.005249 & 0.000000\end{array}$


H. Structure of $p$-Br-phenol in water

$\begin{array}{lrrr}\mathrm{C} & -1.779618 & -1.202040 & -0.000000 \\ \mathrm{C} & -2.473414 & 0.014447 & 0.000000 \\ \mathrm{C} & -1.769074 & 1.224711 & 0.000000 \\ \mathrm{C} & -0.373387 & 1.219417 & -0.000000 \\ \mathrm{C} & 0.309266 & 0.002215 & -0.000000 \\ \mathrm{C} & -0.382235 & -1.208311 & 0.000000 \\ \mathrm{H} & -2.321783 & -2.143774 & 0.000000 \\ \mathrm{H} & -2.315031 & 2.162688 & 0.000000 \\ \mathrm{H} & 0.170571 & 2.157710 & 0.000000 \\ \mathrm{H} & 0.153044 & -2.151538 & 0.000000 \\ \mathrm{O} & -3.840247 & 0.084964 & 0.000000 \\ \mathrm{H} & -4.226390 & -0.802794 & 0.000000 \\ \mathrm{Br} & 2.230638 & -0.005847 & 0.000000\end{array}$

I. Structure of Cl-benzene in gas-phase

$\begin{array}{rrrr}\text { C } & -1.575533 & -1.208858 & 0.000001 \\ \text { C } & -0.177675 & -1.217201 & 0.000008 \\ \text { C } & 0.505749 & -0.000015 & -0.000020 \\ \text { C } & -0.177671 & 1.217196 & -0.000007 \\ \text { C } & -1.575508 & 1.208875 & 0.000017 \\ \text { C } & -2.277379 & 0.000003 & -0.000009 \\ \text { H } & -2.112987 & -2.152793 & 0.000004 \\ \text { H } & 0.374447 & -2.150842 & 0.000008 \\ \text { H } & 0.374496 & 2.150812 & -0.000010 \\ \text { H } & -2.112980 & 2.152798 & 0.000021 \\ \text { H } & -3.363162 & 0.000026 & -0.000014 \\ \text { Cl } & 2.265194 & 0.000000 & 0.000003\end{array}$

J. Structure of Cl-benzene in water

C

$\begin{array}{lll}-0.000000 & -1.219227 & -0.177818\end{array}$ 


$\begin{array}{rrrr}\text { C } & 0.000000 & -0.000000 & 0.501762 \\ \text { C } & 0.000000 & 1.219227 & -0.177818 \\ \text { C } & -0.000000 & 1.210074 & -1.576705 \\ \text { C } & -0.000000 & 0.000000 & -2.278077 \\ \text { C } & -0.000000 & -1.210074 & -1.576705 \\ \text { H } & -0.000000 & -2.154383 & 0.371710 \\ \text { H } & 0.000000 & 2.154383 & 0.371710 \\ H & -0.000000 & 2.154046 & -2.113680 \\ H & -0.000000 & 0.000000 & -3.363713 \\ H & -0.000000 & -2.154046 & -2.113680 \\ \text { Cl } & 0.000000 & -0.000000 & 2.268225\end{array}$




\section{SINGLET-TRIPLET GAPS FOR A SUBSET OF AC12 BENCHMARK SET}

TABLE S1: Computational and experimental S-T gaps of carbene derivatives in acetonitrile. The solvation effects were taken into account by C-PCM, IEF-PCM and RISM models. The cc-pVTZ basis set was used and the 1s core orbitals were treated as frozen at CASPT2 level. The $\pi$ and $\pi^{*}$ orbitals and lone pairs were included in the active space thus leading to $\operatorname{CAS}(8 e, 8 o)$ treatment for phenylcarbene and pentafluorophenylcarbene, $\mathrm{CAS}(10 e, 10 o)$ treatment for diphenylcarbene and fluorenylidene. No thermal corrections are added to each value of $\mathrm{S}-\mathrm{T}$ gap.

\begin{tabular}{|c|c|c|c|c|c|}
\hline & & phenylcarbene & pentafluorophenylcarbene & diphenylcarbene & fluorenylidene \\
\hline \multirow{5}{*}{ Gas Phase } & B3LYP & -5.87 & -5.89 & -5.70 & -5.14 \\
\hline & DLPNO-CCSD $[\mathrm{T} / \mathrm{Q}]$ & -4.83 & -5.91 & -4.52 & -2.92 \\
\hline & $\operatorname{DLPNO}-\mathrm{CCSD}(\mathrm{T})[\mathrm{T} / \mathrm{Q}]$ & -3.87 & -4.49 & -4.41 & -2.48 \\
\hline & CASSCF & -7.34 & -9.33 & -6.30 & -2.10 \\
\hline & CASPT2 & -6.60 & -7.32 & -3.38 & -4.15 \\
\hline \multirow{3}{*}{ C-PCM } & B3LYP & -0.67 & -0.76 & -2.49 & 0.18 \\
\hline & CASSCF & -3.98 & -5.80 & -3.60 & 1.47 \\
\hline & CASPT2 & -2.57 & -3.38 & -0.88 & 0.31 \\
\hline \multirow{3}{*}{ IEF-PCM } & B3LYP & -0.67 & -0.71 & -2.49 & 0.19 \\
\hline & CASSCF & -3.99 & -5.80 & -3.82 & 1.45 \\
\hline & CASPT2 & -2.57 & -3.34 & -0.70 & 0.31 \\
\hline \multirow{4}{*}{ RISM } & B3LYP & -3.00 & -3.57 & -4.56 & -2.42 \\
\hline & CASSCF & -5.52 & -7.93 & -5.10 & -0.21 \\
\hline & CASPT2 & -4.21 & -5.47 & -2.45 & -1.79 \\
\hline & Exp. & -2.30 & -3.10 & -2.80 or -3.40 & -0.20 \\
\hline
\end{tabular}




\section{THE $\left\langle S^{2}\right\rangle$ VALUES OF POLYCARBENE SYSTEMS BY BS-DFT CALCULATIONS}

TABLE S2. The $\left\langle S^{2}\right\rangle$ values of PC1 and PC2 systems by the unrestricted DFT calculations. The singlet states were calculated by broken-symmetry schemes.

\begin{tabular}{ccccc}
\hline \hline & \multicolumn{2}{c}{ PC1 } & PC2 \\
\cline { 2 - 4 } & $S=0$ & $S=1$ & $S=0$ & $S=2$ \\
B3LYP & 1.003 & 2.023 & 1.651 & 6.071 \\
TPSSh & 1.002 & 2.025 & 1.692 & 6.075 \\
& \multicolumn{4}{c}{ C-PCM } \\
B3LYP & 1.004 & 2.029 & 2.042 & 6.082 \\
TPSSh & 1.004 & 2.031 & 2.047 & 6.087 \\
\multicolumn{5}{c}{ IEF-PCM } \\
B3LYP & 1.004 & 2.029 & 2.042 & 6.082 \\
TPSSh & 1.004 & 2.031 & 2.047 & 6.087 \\
\hline
\end{tabular}

\section{STRUCTURES OF POLYCARBENE SYSTEMS}

\section{A. Structure of PC1 system}

$\begin{array}{lrrr}\text { C } & 0.00000000 & 0.00000000 & 0.70419700 \\ \text { C } & 0.00000000 & 1.34380800 & 0.30719900 \\ \text { C } & 0.00000000 & 2.35595800 & 1.30337100 \\ \text { C } & 0.00000000 & 1.76861300 & -1.04898400 \\ \text { C } & 0.00000000 & 3.69506400 & 0.96486700 \\ \text { H } & 0.00000000 & 2.05634900 & 2.34247500 \\ \text { C } & 0.00000000 & 3.11365600 & -1.37132100 \\ \text { H } & 0.00000000 & 1.03588300 & -1.84129700 \\ \text { C } & 0.00000000 & 4.08695900 & -0.37381800 \\ \text { H } & 0.00000000 & 4.44319400 & 1.74688400 \\ \text { H } & 0.00000000 & 3.40912900 & -2.41269300 \\ \text { H } & 0.00000000 & 5.13622900 & -0.63584800 \\ \text { C } & 0.00000000 & -1.34380800 & 0.30719900 \\ \text { C } & 0.00000000 & -2.35595800 & 1.30337100 \\ \text { C } & 0.00000000 & -1.76861300 & -1.04898400 \\ \text { C } & 0.00000000 & -3.69506400 & 0.96486700\end{array}$




$\begin{array}{lrrr}\mathrm{H} & 0.00000000 & -2.05634900 & 2.34247500 \\ \mathrm{C} & 0.00000000 & -3.11365600 & -1.37132100 \\ \mathrm{H} & 0.00000000 & -1.03588300 & -1.84129700 \\ \mathrm{C} & 0.00000000 & -4.08695900 & -0.37381800 \\ \mathrm{H} & 0.00000000 & -4.44319400 & 1.74688400 \\ \mathrm{H} & 0.00000000 & -3.40912900 & -2.41269300 \\ \mathrm{H} & 0.00000000 & -5.13622900 & -0.63584800\end{array}$

B. Structure of PC2 system

\begin{tabular}{|c|c|c|c|}
\hline $\mathrm{C}$ & 0.00000000 & 2.41939900 & -0.42825600 \\
\hline $\mathrm{C}$ & 0.00000000 & 3.82258300 & -0.41717900 \\
\hline $\mathrm{C}$ & 0.00000000 & 4.51809200 & -1.65410400 \\
\hline $\mathrm{C}$ & 0.00000000 & 4.60543400 & 0.76774100 \\
\hline $\mathrm{C}$ & 0.00000000 & 5.89906300 & -1.70014300 \\
\hline $\mathrm{H}$ & 0.00000000 & 3.94232000 & -2.56950200 \\
\hline $\mathrm{C}$ & 0.00000000 & 5.98732300 & 0.70526500 \\
\hline $\mathrm{H}$ & 0.00000000 & 4.12092100 & 1.73208600 \\
\hline $\mathrm{C}$ & 0.00000000 & 6.64607900 & -0.52276400 \\
\hline $\mathrm{H}$ & 0.00000000 & 6.40105400 & -2.65888800 \\
\hline $\mathrm{H}$ & 0.00000000 & 6.55959200 & 1.62407800 \\
\hline $\mathrm{H}$ & 0.00000000 & 7.72690000 & -0.56153600 \\
\hline $\mathrm{C}$ & 0.00000000 & 1.23924100 & 0.32004800 \\
\hline $\mathrm{C}$ & 0.00000000 & 0.00000000 & -0.35795100 \\
\hline $\mathrm{C}$ & 0.00000000 & 1.21016400 & 1.74649800 \\
\hline $\mathrm{C}$ & 0.00000000 & -1.23924100 & 0.32004800 \\
\hline $\mathrm{H}$ & 0.00000000 & 0.00000000 & -1.43867700 \\
\hline $\mathrm{C}$ & 0.00000000 & 0.00000000 & 2.42174400 \\
\hline $\mathrm{H}$ & 0.00000000 & 2.13178800 & 2.30682100 \\
\hline $\mathrm{C}$ & 0.00000000 & -1.21016400 & 1.74649800 \\
\hline $\mathrm{H}$ & 0.00000000 & 0.00000000 & 3.50451000 \\
\hline $\mathrm{H}$ & 0.00000000 & -2.13178800 & 2.30682100 \\
\hline $\mathrm{C}$ & 0.00000000 & -2.41939900 & -0.42825600 \\
\hline
\end{tabular}


C

C

C

C

$\mathrm{H}$

C

$\mathrm{H}$

C

$\mathrm{H}$

$\mathrm{H}$

$\mathrm{H}$
$0.00000000-3.82258300-0.41717900$

$\begin{array}{lll}0.00000000 & -4.51809200 & -1.65410400\end{array}$

$\begin{array}{lll}0.00000000 & -4.60543400 & 0.76774100\end{array}$

$\begin{array}{lll}0.00000000 & -5.89906300 & -1.70014300\end{array}$

$0.00000000-3.94232000-2.56950200$

$\begin{array}{lll}0.00000000 & -5.98732300 & 0.70526500\end{array}$

$\begin{array}{lll}0.00000000 & -4.12092100 & 1.73208600\end{array}$

$\begin{array}{lll}0.00000000 & -6.64607900 & -0.52276400\end{array}$

$0.00000000-6.40105400-2.65888800$

$0.00000000 \quad-6.55959200 \quad 1.62407800$

$0.00000000 \quad-7.72690000 \quad-0.56153600$

\section{BENCHMARK RESULTS INCLUDING IEF-PCM VALUES}


TABLE S3: Solvation free energies (in $\mathrm{kcal} / \mathrm{mol}$ ) calculated by several PCM-based methods including PCM-CASPT2 in

comparison with the experimental values. The $6-31+\mathrm{G}^{* *}$ basis set was used and 1s core orbitals were treated as frozen at

CASPT2 level. However, 6-31G** basis set was selected for Br.

The $\pi$ and $\pi^{*}$ orbitals were included in the active space thus

leading to $\mathrm{CAS}(6 e, 6 o)$ treatment. Thermal corrections for

C-PCM, IEF-PCM and RISM calculations were calculated at DFT level of theory using GAMESS-US package.

\begin{tabular}{|c|c|c|c|c|c|c|c|c|c|}
\hline & \multicolumn{4}{|c|}{ C-PCM } & \multicolumn{3}{|c|}{ IEF-PCM } & \multirow[b]{2}{*}{ CASPT2 } & \multirow[b]{2}{*}{ Exp. } \\
\hline & B3LYP & CASSCF & $\delta \sigma$ & CASPT2 & B3LYP & CASSCF & $\delta \sigma$ & & \\
\hline Pyridine & -4.63 & -4.73 & $(-0.021)$ & -4.83 & -4.60 & -4.69 & $(-0.021)$ & -4.79 & -4.70 \\
\hline Aniline & -5.69 & -6.05 & $(-0.024)$ & -5.84 & -5.69 & -6.03 & $(-0.024)$ & -5.83 & -5.50 \\
\hline Phenol & -5.77 & -6.36 & $(-0.021)$ & -5.96 & -5.80 & -6.40 & $(-0.022)$ & -6.00 & -6.60 \\
\hline p-Br-phenol & -6.28 & -6.98 & $(-0.024)$ & -6.36 & -6.39 & -7.11 & $(-0.027)$ & -6.47 & -7.13 \\
\hline Cl-benzene & -2.65 & -3.02 & $(-0.026)$ & -2.53 & -2.68 & -3.05 & $(-0.027)$ & -2.56 & -1.12 \\
\hline
\end{tabular}


TABLE S4: Computational and experimental isomerization energies of several organic moelcules in water. The solvation effects were taken into account by C-PCM, IEF-PCM and RISM models. The maug-cc-pVTZ basis set was used and 1s core orbitals were treated as frozen at CASPT2 level. The $\pi$ and $\pi^{*}$ orbitals were included in the active space thus leading to $\operatorname{CAS}(5 e, 6 o)$ treatment for 1,2,3-triazole, $\operatorname{CAS}(8 e, 8 o)$ treatment for isonicotinic acid, and $\operatorname{CAS}(8 e, 10 o)$ treatment for cytosin and 6-chloro-2-pyridone. Thermal corrections for C-PCM, IEF-PCM and RISM calculations were calculated at DFT level of theory using GAMESS-US package.

\begin{tabular}{|c|c|c|c|c|c|c|}
\hline & & $\begin{array}{c}\text { 1,2,3-triazole } \\
1 \mathrm{H} \rightarrow 2 \mathrm{H}\end{array}$ & $\begin{array}{c}\text { isonicotinic acid } \\
\qquad \mathrm{N} \rightarrow \mathrm{Z}\end{array}$ & $\begin{array}{l}\text { cytosine } \\
\mathrm{C} 1 \rightarrow \mathrm{C} 3\end{array}$ & $\begin{array}{l}\text { cytosine } \\
\mathrm{C} 1 \rightarrow \mathrm{C} 8\end{array}$ & $\begin{array}{c}\text { 6-chloro-2-pyridone } \\
\qquad \mathrm{K} \rightarrow \mathrm{E}\end{array}$ \\
\hline \multirow{4}{*}{ Gas Phase } & B3LYP & -3.73 & 29.76 & 7.29 & 0.70 & -2.80 \\
\hline & $\operatorname{CCSD}(\mathrm{T})$ & -3.59 & 32.65 & 7.00 & 1.00 & -3.92 \\
\hline & CASSCF & -4.22 & 52.48 & 9.29 & -5.19 & -2.79 \\
\hline & CASPT2 & -4.22 & 31.39 & 7.53 & -0.05 & -5.36 \\
\hline \multirow{3}{*}{ C-PCM } & B3LYP & -0.28 & 0.72 & 4.51 & 5.65 & 1.30 \\
\hline & CASSCF & -1.01 & 16.03 & 7.33 & -0.15 & -0.43 \\
\hline & CASPT2 & -0.51 & 1.26 & 3.65 & 5.84 & -1.82 \\
\hline \multirow{5}{*}{ IEF-PCM } & B3LYP & -0.31 & 0.81 & 4.33 & 5.89 & 1.48 \\
\hline & $\operatorname{CCSD}(\mathrm{T})^{a}$ & -0.68 & 8.15 & 5.26 & 4.22 & -1.47 \\
\hline & $\operatorname{CCSD}(\mathrm{T})^{b}$ & -0.85 & 2.35 & 4.32 & 7.04 & -2.30 \\
\hline & CASSCF & -1.04 & 16.14 & 7.17 & -0.23 & -0.29 \\
\hline & CASPT2 & -0.54 & 1.35 & 3.45 & 5.77 & -1.64 \\
\hline \multirow{5}{*}{ RISM } & B3LYP & -0.41 & -2.66 & 3.79 & 7.09 & 1.57 \\
\hline & $\operatorname{CCSD}(\mathrm{T})$ & -0.30 & -1.46 & 3.33 & 6.00 & 0.53 \\
\hline & CASSCF & 0.10 & 9.43 & 7.39 & 4.08 & 0.16 \\
\hline & CASPT2 & 0.65 & -4.58 & 2.92 & 10.10 & -1.48 \\
\hline & Exp. & $-0.38,-2.45$ & -2.44 to -2.59 & 3.60 & 5.50 to 6.90 & 0.46 \\
\hline
\end{tabular}

${ }^{a}$ PTE-PCM scheme for solvation

${ }^{b}$ Values taken from Ref. 1 
TABLE S5: Computational and experimental S-T gaps of carbene derivatives in acetonitrile. The solvation effects were taken into account by C-PCM, IEF-PCM and RISM models. The cc-pVTZ basis set was used and the 1s core orbitals were treated as frozen at CASPT2 level. The $\pi$ and $\pi^{*}$ orbitals and lone pairs were included in the active space thus leading to $\operatorname{CAS}(8 e, 8 o)$ treatment for phenylcarbene and pentafluorophenylcarbene, $\mathrm{CAS}(10 e, 10 o)$ treatment for diphenylcarbene and fluorenylidene. Thermal corrections for C-PCM, IEF-PCM and RISM calculations were calculated at DFT level of theory using Gaussian 16 package.

\begin{tabular}{|c|c|c|c|c|c|}
\hline & & phenylcarbene & pentafluorophenylcarbene & diphenylcarbene & fluorenylidene \\
\hline & B3LYP & -6.73 & -6.92 & -7.41 & -5.34 \\
\hline & DLPNO-CCSD $[\mathrm{T} / \mathrm{Q}]$ & -4.83 & -5.91 & -4.52 & -2.92 \\
\hline \multirow[t]{4}{*}{ Gas Phase } & $\operatorname{DLPNO}-\mathrm{CCSD}(\mathrm{T})[\mathrm{T} / \mathrm{Q}]$ & -3.87 & -4.49 & -4.41 & -2.48 \\
\hline & CASSCF & -8.21 & -10.35 & -8.01 & -2.30 \\
\hline & CASPT2 & -7.47 & -8.34 & -5.09 & -4.35 \\
\hline & B3LYP & -1.92 & -2.18 & -4.33 & -0.38 \\
\hline \multirow[t]{3}{*}{ C-PCM } & CASSCF & -5.23 & -7.22 & -5.44 & 0.91 \\
\hline & CASPT2 & -3.81 & -4.80 & -2.72 & -0.25 \\
\hline & B3LYP & -1.92 & -2.13 & -4.33 & -0.37 \\
\hline \multirow[t]{3}{*}{ IEF-PCM } & CASSCF & -5.23 & -7.22 & -5.67 & 0.89 \\
\hline & CASPT2 & -3.81 & -4.76 & -2.55 & -0.25 \\
\hline & B3LYP & -4.25 & -4.99 & -6.40 & -2.98 \\
\hline \multirow[t]{3}{*}{ RISM } & CASSCF & -6.76 & -9.36 & -6.95 & -0.77 \\
\hline & CASPT2 & -5.45 & -6.90 & -4.29 & -2.35 \\
\hline & Exp. & -2.30 & -3.10 & -2.80 or -3.40 & -0.20 \\
\hline
\end{tabular}


TABLE S6: The computational S-T and S-Q gaps of N-doped polycarbenes in acetonitrile solvent at the DMRG-CASSCF/CASPT2 level. The solvation effects were taken into account by the C-PCM, IEF-PCM and RISM models. The def2-SVP basis set was used and 1s core orbitals of $\mathrm{C}$ and $\mathrm{N}$ atoms were treated as frozen at the CASPT2 level. The $\pi$ and $\pi^{*}$ orbitals and lone pairs were included in the active space thus leading to $\operatorname{CAS}(14 e, 14 o)$ and $\mathrm{CAS}(22 e, 22 o)$ treatments for $\mathrm{PC} 1$ and $\mathrm{PC} 2$, respectively. The energy gaps calculated for the closed-shell and the broken-symmetry singlet states are indicated with "CS" and "BS", respectively. The triplet state for the $\mathrm{PC} 1$ and quintet state for the $\mathrm{PC} 2$ are dominated by the high-spin open-shell configurations. The difference betwen the norm of the dipole moments for the final high-spin and the initial siglet states is also shown at the DFT and the CASSCF levels.

\begin{tabular}{|c|c|c|c|c|c|}
\hline & & \multicolumn{2}{|l|}{$\mathrm{PC} 1$} & \multicolumn{2}{|c|}{$\mathrm{PC} 2$} \\
\hline & & $\Delta \mathrm{E}_{\mathrm{S}-\mathrm{T}}(\mathrm{kcal} / \mathrm{mol})$ & $\Delta\|\mu\|_{\mathrm{S}-\mathrm{T}}(\mathrm{D})$ & $\Delta \mathrm{E}_{\mathrm{S}-\mathrm{Q}}(\mathrm{kcal} / \mathrm{mol})$ & $\Delta\|\mu\|_{\mathrm{S}-\mathrm{Q}}(\mathrm{D})$ \\
\hline \multirow{6}{*}{ Gas-phase } & B3LYP (BS) & -13.20 & -0.42 & -0.58 & 8.33 \\
\hline & B3LYP (CS) & -34.37 & 0.70 & -37.01 & 11.06 \\
\hline & TPSSh (BS) & -14.33 & -0.32 & -0.43 & 7.65 \\
\hline & TPSSh (CS) & -51.97 & 1.76 & -38.04 & 11.69 \\
\hline & CASSCF & -16.37 & 2.15 & -6.46 & 0.15 \\
\hline & CASPT2 & -11.51 & - & -4.76 & - \\
\hline \multirow{6}{*}{ C-PCM } & B3LYP (BS) & -12.81 & -0.34 & -4.10 & 0.15 \\
\hline & B3LYP (CS) & -33.52 & 1.06 & -44.18 & 14.04 \\
\hline & TPSSh (BS) & -14.03 & -0.21 & -4.06 & 0.17 \\
\hline & TPSSh (CS) & -40.16 & 0.44 & -45.56 & 15.33 \\
\hline & CASSCF & -18.80 & 1.22 & -6.64 & 0.07 \\
\hline & CASPT2 & -14.98 & - & -4.95 & - \\
\hline \multirow{6}{*}{ IEF-PCM } & B3LYP (BS) & -12.81 & -0.34 & -4.10 & 0.15 \\
\hline & B3LYP (CS) & -33.55 & 1.06 & -44.16 & 14.04 \\
\hline & TPSSh (BS) & -14.03 & -0.22 & -4.06 & 0.17 \\
\hline & TPSSh (CS) & -43.90 & 0.45 & -45.53 & 15.32 \\
\hline & CASSCF & -18.80 & 1.22 & -6.63 & 0.07 \\
\hline & CASPT2 & -14.99 & - & -4.94 & - \\
\hline RISM & CASSCF & -18.36 & 1.49 & -6.60 & 0.09 \\
\hline
\end{tabular}


[1] D. Yokogawa, Journal of Chemical Theory and Computation 14, 2661 (2018), pMID: 29595975, https://doi.org/10.1021/acs.jctc.8b00168. 